\title{
Dual Regulation of Anterograde and Retrograde Transmission by Endocannabinoids
}

\author{
Karl J. Iremonger, J. Brent Kuzmiski, Dinara V. Baimoukhametova, and Jaideep S. Bains \\ Hotchkiss Brain Institute and the Department of Physiology and Pharmacology, University of Calgary, Calgary, Alberta T2N 4N1, Canada
}

Endocannabinoids (eCBs) are feedback messengers in the nervous system that act at the presynaptic nerve terminal to inhibit transmitter release. Here we report that in brain slices from rat, $\mathrm{eCBs}$ are released from vasopressin (VP) neurons in the paraventricular nucleus of the hypothalamus following coincident bursts of presynaptic and postsynaptic spiking. eCBs transiently depress glutamate release from excitatory terminals and, in doing so, prevent the synapses from undergoing long-term depression (LTD). Specifically, we show that blockade of CB1 receptors unmasks LTD following coincident presynaptic and postsynaptic activity. This LTD is presynaptic in nature, but requires the release of the opioid peptide dynorphin from the postsynaptic neuron. Dynorphin release and subsequent LTD require the activation of postsynaptic metabotropic glutamate receptors (mGluRs). Our findings indicate that eCBs, by transiently depressing glutamate release, limit mGluR activation and indirectly gate release of dynorphin from the postsynaptic neuron. We propose that eCBs, in addition to their well described role in the rapid modulation of transmitter release from the nerve terminal, also regulate the release of other retrograde transmitters and thus encode for multiple temporal windows of synaptic plasticity.

\section{Introduction}

In addition to releasing transmitters from their nerve terminals, neurons also release messengers from their somatodendritic compartments. These messengers function as retrograde signals to regulate the strength of afferent input to the postsynaptic neuron (Regehr et al., 2009). Numerous molecules, including lipid precursors, amino acids, and neuropeptides, have been identified as retrograde transmitters, raising the intriguing possibility that multiple retrograde signals originate from a single postsynaptic neuron. While the concept of anterograde cotransmission is widely accepted (Kupfermann, 1991; Weisskopf et al., 1993; Jonas et al., 1998; Jo and Schlichter, 1999; Lu et al., 2008; Tecuapetla et al., 2010), there are only a few examples of neurons coreleasing retrograde messengers (Duguid and Smart, 2004; Hirasawa et al., 2004; Zilberter et al., 2005; Di et al., 2009). Consequently, little is known about the specific conditions that would bias a neuron toward the release of a specific retrograde signal, what consequence each signal would have on synaptic strength, and what impact the release of one signal would have on the subsequent release of different signals.

Received June 10, 2011; revised June 27, 2011; accepted June 28, 2011.

Author contributions: K.J.I. and J.S.B. designed research; K.J.I., J.B.K., and D.V.B. performed research; K.J.I., J.B.K., and J.S.B. analyzed data; K.J.I. and J.S.B. wrote the paper.

This work was supported by operating grants from the Canadian Institutes of Health Research (J.S.B.) and the Heart and Stroke Foundation of Alberta, Yukon, and Northwest Territories (J.S.B.). K.J.I. was supported by studentships from the Heart and Stroke Foundation of Canada and the Alberta Heritage Foundation for Medical Research (AHFMR). J.S.B. is an AHFMR Senior Scholar. We thank Dr. R. Piet for comments on this manuscript and members of the Bains laboratory for fruitful discussions.

Correspondence should be addressed to Jaideep S. Bains, Hotchkiss Brain Institute and the Department of Physiology and Pharmacology, University of Calgary, 3330 Hospital Drive NW, Calgary, AB T2N 4N1, Canada. E-mail: jsbains@ucalgary.ca.

K. J. Iremonger's present address: Center for Neuroendocrinology, Department of Physiology, University of Otago, P.0. Box 913, Dunedin 9054, New Zealand.

DOI:10.1523/JNEUROSCI.2925-11.2011

Copyright $\odot 2011$ the authors $\quad 0270-6474 / 11 / 3112011-10 \$ 15.00 / 0$
We have previously demonstrated that vasopressin (VP)producing magnocellular neurosecretory cells (MNCs) in the paraventricular nucleus of the hypothalamus (PVN) release dynorphin, which acts as a retrograde transmitter to produce a long-term depression (LTD) of excitatory glutamate transmission (Iremonger and Bains, 2009). MNCs, however, are also capable of releasing other molecules from their somatodendritic compartment, including nitric oxide, neuropeptides, and endocannabinoids (eCBs) (Bains and Ferguson, 1997; Brown and Bourque, 2004; Hirasawa et al., 2004; Ludwig et al., 2005; Di et al., 2009). In this current study, we asked whether physiologically relevant bursts of spiking activity either in the absence of presence of synaptic activity, could drive the release of multiple retrograde transmitters from a single neuron and whether these different retrograde signaling molecules interact with one another to regulate synaptic strength.

Here we show that bursts of presynaptic activity paired with postsynaptic spiking result in the release of two different retrograde transmitters, eCBs and dynorphin. There is a dynamic interaction between these different retrograde channels, so that release of one makes the release of a second less likely. Specifically, eCBs depress glutamate release from presynaptic terminals, thus reducing the activation of postsynaptic metabotropic glutamate receptors (mGluRs) and subsequent release of dynorphin. Relief of this eCB-mediated inhibition allows for robust dynorphin release and unmasks an LTD of glutamate transmission. These findings indicate that eCBs, by regulating both anterograde and retrograde neurotransmission at glutamatergic synapses onto VP neurons, control both the magnitude and the duration of presynaptic depression.

\section{Materials and Methods}

All protocols were approved by the University of Calgary Animal Care and Use Committee in accordance with guidelines established by the 
Canadian Council on Animal Care. Male Wistar/Sprague Dawley (p21-p40) rats that express enhanced green fluorescence protein (eGFP) under the VP promoter were used (Ueta et al., 2005). Animals were anesthetized with sodium pentobarbital $(0.1 \mathrm{ml} / 100 \mathrm{~g}$ of body weight) and then decapitated. The brain was quickly removed and placed in ice-cold slicing solution for several minutes containing the following (in $\mathrm{mm}$ ): $87 \mathrm{NaCl}, 2.5 \mathrm{KCl}, 25$ $\mathrm{NaHCO}_{3}, 0.5 \mathrm{CaCl}_{2}, 7 \mathrm{MgCl}_{2}, 1.25 \mathrm{NaH}_{2} \mathrm{PO}_{4}$, 25 glucose, 75 sucrose; saturated with $95 \%$ $\mathrm{O}_{2} / 5 \% \mathrm{CO}_{2}$. The brain was then blocked and mounted on a vibrating slicer (Leica) submerged in ice-cold slicing solution. Slices were incubated at $32.5^{\circ} \mathrm{C}$ in artificial CSF (ACSF) containing the following (in $\mathrm{mM}$ ): $126 \mathrm{NaCl}$, $2.5 \mathrm{KCl}, 26 \mathrm{NaHCO}_{3}, 2.5 \mathrm{CaCl}_{2}, 1.5 \mathrm{MgCl}_{2}$, $1.25 \mathrm{NaH}_{2} \mathrm{PO}_{4}, 10$ glucose; saturated with $95 \%$ $\mathrm{O}_{2} / 5 \% \mathrm{CO}_{2}$, for a minimum of $60 \mathrm{~min}$.

Electrophysiology. Slices containing the PVN were submerged in a recording chamber and superfused with $32.5^{\circ} \mathrm{C} \mathrm{ACSF}$ at a flow rate of 1 $\mathrm{ml} / \mathrm{min}$. Whole-cell recordings were obtained from VP MNCs visualized with an AxioskopII FS Plus (Zeiss) upright microscope fitted with infrared differential interference contrast optics. Recorded cells were confirmed to be MNCs based on their morphology and well defined electrophysiological characteristics ( $\mathrm{Lu}-$ ther and Tasker, 2000). eGFP neurons were visualized with a Zeiss AxioCam MRm camera.

Patch pipettes were pulled from borosilicate glass, had a resistance between 3 and $6 \mathrm{M} \Omega$ and were filled with a solution containing the following (in $\mathrm{mm}$ ): 116 potassium gluconate, 8 sodium gluconate, $2 \mathrm{MgCl}_{2}, 8 \mathrm{KCl}, 0.1$ potassium EGTA, 4 potassium ATP, and 0.3 sodium GTP, 10 HEPES, corrected to $\mathrm{pH} 7.2$ with KOH. Series resistance was not compensated and recordings were accepted for analysis if changes in access resistance were $<20 \%$. The liquid junction potential was calculated to be approximately $-13 \mathrm{mV}$ and was not compensated for.

For voltage-clamp experiments, cells were held at $-60 \mathrm{mV}$. For currentclamp experiments, in between periods of stimulation, cells were held around $-60 \mathrm{mV}$. This was to ensure consistency across experimental conditions.

Glutamatergic fibers were stimulated extracellularly with a monopolar glass microelectrode (3-6 M $\Omega$ ) filled with ACSF and placed either within or just outside of the PVN. Synaptic stimulation was delivered at a rate of $0.2 \mathrm{~Hz}$ for all experiments unless otherwise stated. Each data point in most summary graphs represents the mean ( \pm SEM) of 15 EPSCs $(1$ block of trials). Each block of trials commenced 2 min after the beginning of the previous block.

The perfusate always contained picrotoxin $(100 \mu \mathrm{M})$ to block $\mathrm{GABA}_{\mathrm{A}^{-}}$ mediated conductances. Drugs used were purchased from the following locations: $\quad N$-(piperidin-1-yl)-5-(4-iodophenyl)-1-(2,4-dichlorophenyl)4-methyl-1 H-pyrazole-3-carboxamide (AM251): Tocris Bioscience; dynorphin A(1-17): Bachem. All remaining drugs and chemicals were purchased from Sigma-Aldrich.

Data collection and analysis. Signals were amplified with the Multiclamp 700B amplifier (Molecular Devices), were low-pass filtered at 1 $\mathrm{kHz}$, digitized at $10-20 \mathrm{kHz}$ with the Digidata 1322 (Molecular Devices), and saved on a PC for offline analysis. For evoked currents, analysis was performed using pClamp 9 (Molecular Devices). To better quantify synaptic efficacy at these synapses that exhibit pronounced asynchronous release, we measured charge transfer rather than peak amplitude. For all experiments, charge transfer was calculated by integrating the area under the averaged evoked EPSC from the onset of the EPSC to $100 \mathrm{~ms}$. This
B

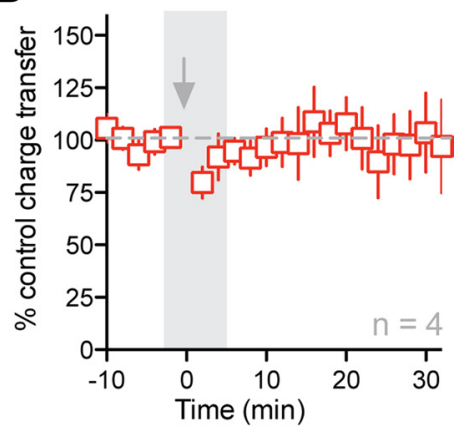

D

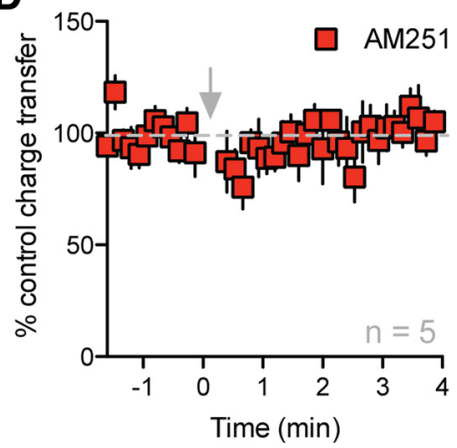

time window generally encompasses all asynchronous release events (Iremonger and Bains, 2007).

The total number of individual asynchronous release events was quantified by counting the occurrence of sEPSCs from 5 to $100 \mathrm{~ms}$ after the onset of the EPSC across 15 trials. Events were not counted in the first 5 $\mathrm{m}$ because they could not be discriminated from the synchronous component. During the 5-10 ms time window (decay of the synchronous EPSC), we could clearly discern individual quanta, and these were counted as asynchronous events. Baseline spontaneous release was calculated for the $100 \mathrm{~ms}$ before the stimulus and subtracted. For each cell, the total number of asynchronous events was compared across each time point. For amplitude analysis of asynchronous events, only events with a clear baseline before onset were selected, and those that were summed onto other events were rejected from analysis.

All data are presented as mean \pm SEM. Nonparametric statistical analyses were performed with the Mann-Whitney test when comparing two groups or with the Friedman test with Dunn's multiple comparison test for more than two groups (or multiple time points). $p<0.05$ was accepted as statistically significant $\left({ }^{\star} p<0.05\right)$. For statistical analysis of synaptic depression, EPSC charge was compared between five time points: control, $0 \mathrm{~min}, 10 \mathrm{~min}, 20 \mathrm{~min}$, and $30 \mathrm{~min}$.

\section{Results}

The activity pattern displayed by many VP neurons in vivo is characterized by bursts of spiking $(\sim 10 \mathrm{~Hz})$ for tens of seconds interspersed with equal periods of quiescence. This firing pattern has been termed phasic bursting and requires excitatory synaptic drive in vivo (Nissen et al., 1995; Brown et al., 2004). Since VP neurons are known to express several retrograde transmitters, we tested whether different combinations of presynaptic and post- 

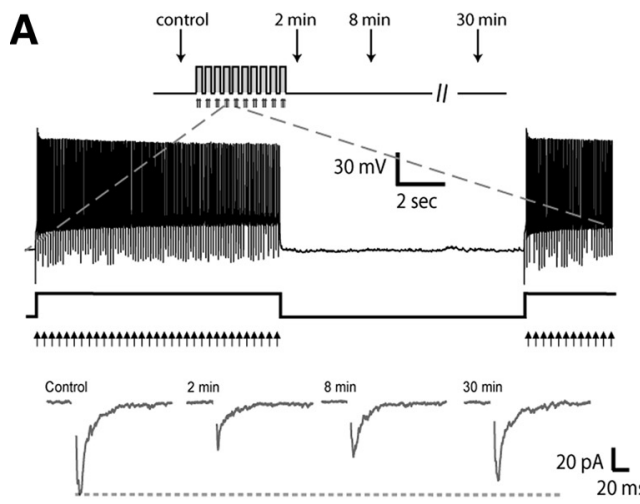

B Spiking paired with synaptic stim

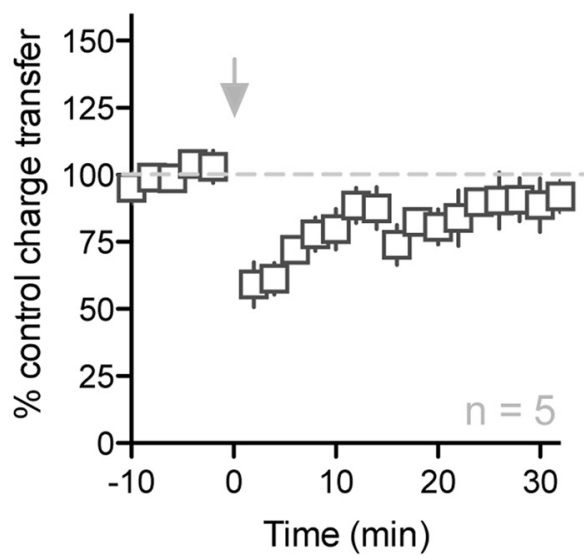

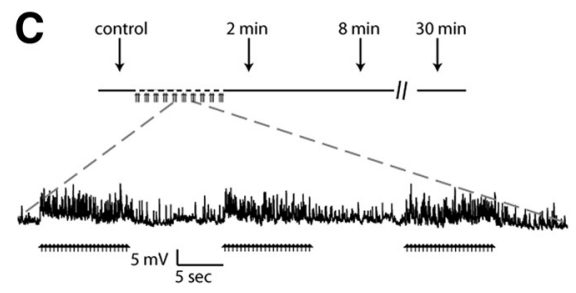

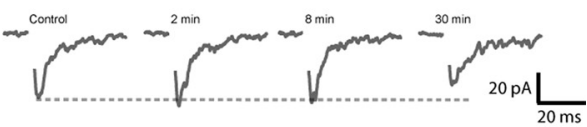

D $\quad 10 \mathrm{~Hz}$ synaptic stim alone

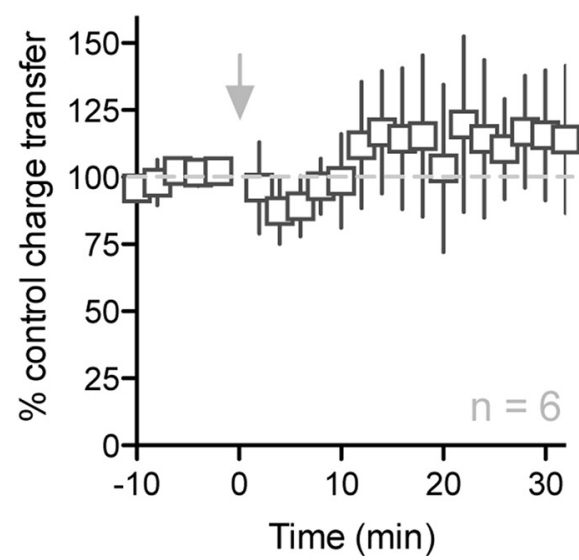

Figure 2. Pairing of synaptic activity and postsynaptic spiking induces a robust synaptic depression. $A$, Synaptic stimulation at $10 \mathrm{~Hz}$ for $10 \mathrm{~s}$ was paired with postsynaptic depolarization to evoke repetitive spiking (average spike frequency: $15.0 \pm 1.7 \mathrm{~Hz}, n=5$ ). This pairing was repeated 10 times with 10 s interburst interval. $\boldsymbol{B}$, After burst pairing, there was a large synaptic depression (EPSC at $0 \mathrm{~min}=59 \pm 8 \%$ of control, $p<0.05, n=5$ ) that recovered back to baseline by $30 \mathrm{~min}(92 \pm 6 \%$ control, $p>0.05, n=5)$. C, Bursts of synaptic stimulation alone ( $10 \times 10 \mathrm{~Hz}$ bursts) did not elicit synaptic depression (EPSC at $0 \mathrm{~min}=96 \pm 16 \%$ of control; EPSC at $30 \mathrm{~min}=114 \pm 28 \%$ of control, $p>0.05, n=6, \boldsymbol{D}$ ).

synaptic bursting activity differentially recruited these retrograde signaling pathways.

In response to a single presynaptic action potential, glutamatergic synapses onto VP neurons often exhibit asynchronous release, which acts to increase the temporal window of excitation (Iremonger and Bains, 2007). Consequently, to assess the impact of retrograde transmitters on synaptic transmission, we measure synaptic strength by taking the integral of the entire evoked EPSC (synaptic charge transfer). This measure encompasses both synchronous and asynchronous components of transmitter release.

\section{Small, transient synaptic depression is induced by postsynaptic spiking alone}

We have previously demonstrated that repetitive postsynaptic depolarizations in the presence of $\mathrm{CB} 1$ receptor $(\mathrm{CB} 1 \mathrm{R})$ blockade and aminopeptidase inhibitors cause an LTD of glutamate transmission that is due to the presynaptic actions of dynorphin, which is released from the dendrites of VP neurons (Iremonger and Bains, 2009). Here, we sought to examine the relationship between eCBs and dynorphin and asked whether specific patterns of activity would bias VP neurons toward releasing one messenger or the other. Brief postsynaptic depolarizations or bursts of spikes can release eCBs. Consequently, we wanted to determine the effects of postsynaptic spiking alone on synaptic strength. Synaptic efficacy was evaluated for $10 \mathrm{~min}$ before and for $30 \mathrm{~min}$ following the spiking protocol by measuring synaptic charge transfer (see methods). The spiking protocol consisted of $10 \mathrm{~s}$ periods of spiking (in current clamp) at a frequency of $10-20 \mathrm{~Hz}$ repeated 10 times with a $10 \mathrm{~s}$ interval between bursts (Fig. $1 \mathrm{~A}$ ). This protocol induced a mean intraburst spike rate of $12.2 \pm 2.3$ $\mathrm{Hz}(n=4)$. Postsynaptic spiking alone failed to induce changes in synaptic efficacy when assessed $30 \mathrm{~min}$ after induction (EPSC at $30 \mathrm{~min}=97 \pm 22 \%$ of control, $p>0.05, n=4$ ) (Fig. $1 \mathrm{~B}$ ). We did, however, observe a small, transient depression of the EPSC immediately following the induction protocol. Analyzing EPSCs at time points immediately after the end of the spiking protocol (without within trial averaging) revealed a brief synaptic depression (charge transfer of first EPSC after spiking $=36 \pm$ $12 \%$ of control, $p<0.05, n=4$ ) (Fig. 1C). This depression was entirely blocked by the CB1 receptor antagonist, AM-251, 5 $\mu \mathrm{M}$ (first EPSC after spiking $=87 \pm 14 \%$ of control, $p>0.05$, $n=5$ ) (Fig. 1D).

These data suggest that postsynaptic spiking alone is sufficient to liberate eCBs and transiently depress glutamate signaling, but under these conditions, we see no evidence for presynaptic depression mediated by dynorphin.

\section{Pairing of presynaptic and postsynaptic bursting evokes robust synaptic depression}

Under some conditions, efficient retrograde transmitter release requires the coincident activation of both postsynaptic glutamate receptors in addition to postsynaptic $\mathrm{Ca}^{2+}$ rises (Varma et al., 2001; Maejima et al., 2005). Therefore we next examined the 
A

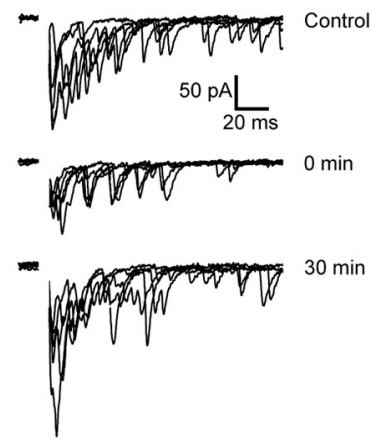

C

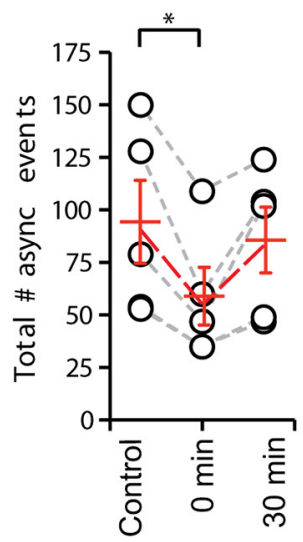

E

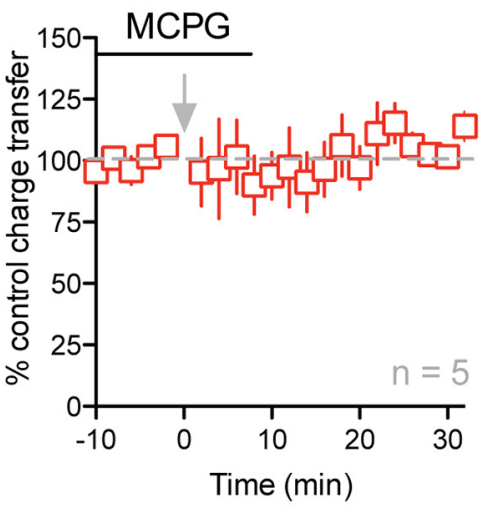

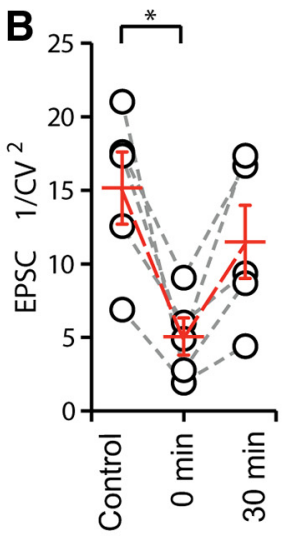

D

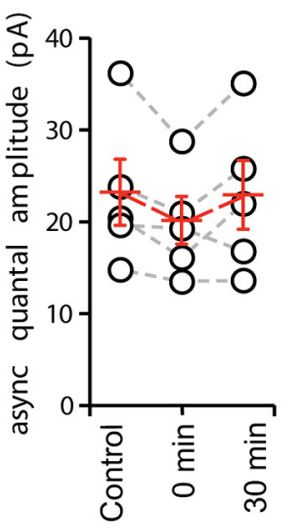

$\mathbf{F}$

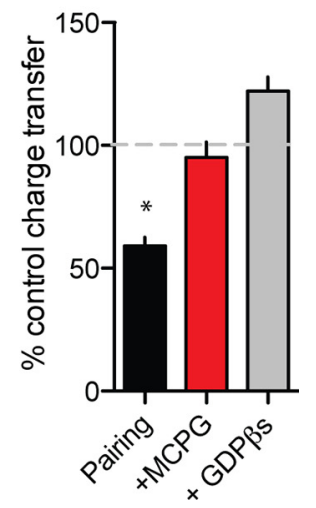

Figure 3. Synaptic depression is presynaptic and requires the activation of postsynaptic mGluRs. $\boldsymbol{A}$, Representative traces (5 sweeps overlaid) from control, immediately after burst pairing ( $0 \mathrm{~min}$ ) and $30 \mathrm{~min}$ after pairing. $\boldsymbol{B}$, There is a significant reduction in $1 / \mathrm{CV}^{2}$ after burst pairing $(p<0.05, n=5)$ that returns to control levels after $30 \mathrm{~min}(p>0.05, n=5)$. C, There is also a significant decrease in the number of asynchronously released quanta ( $62 \pm 4 \%$ of control at 0 min, $p<0.05, n=5$ ) without a significant change in the quantal amplitude ( $87 \pm$ $4 \%$ of control amplitude at $0 \mathrm{~min}, p>0.05, n=5, \boldsymbol{D})$. $\boldsymbol{E}$, Burst pairing in the presence of the group I/II mGluR antagonist MCPG $(200 \mu \mathrm{m})$ completely eliminates the synaptic depression (EPSC at 0 min $=95 \pm 14 \%$ control, $p>0.05, n=5$ ). $\boldsymbol{F}$, Pairing of $10 \mathrm{~Hz}$ synaptic activity with spiking after cells were dialyzed with an internal solution containing $2 \mathrm{~mm} \mathrm{GDP-} \beta$ s also prevented synaptic depression (EPSC at 0 min $=122 \pm 13 \%$ control, $p>0.05, n=5$ ). ${ }^{*}$ denotes $p<0.05$.

effects of bursts of presynaptic stimulation paired with postsynaptic activity on EPSC strength.

Burst pairing consisted of the following protocol: $10 \mathrm{~Hz}$ synaptic stimulation paired with postsynaptic depolarization (cur-

rent injection through the patch pipette) to induce $10-20 \mathrm{~Hz}$ spiking for $10 \mathrm{~s}$ (Fig. $2 \mathrm{~A}$ ). Identical to the protocol used above, bursts were induced 10 times with $10 \mathrm{~s}$ interburst intervals. The mean spike frequency induced during the $10 \mathrm{~s}$ burst was $15.0 \pm 1.7 \mathrm{~Hz}(n=5)$ and was not significantly different $(p>$ $0.05)$ to the firing rate induced by current injection alone in Figure $1 A$. Following the burst pairing protocol, we reliably observed a robust synaptic depression (EPSC at $0 \mathrm{~min}=59 \pm$ $8 \%$ of control, $p<0.05, n=5$ ) (Fig. $2 B$ ), with the EPSC charge transfer returning to control levels after $30 \mathrm{~min}(92 \pm 6 \%$ control, $p>0.05, n=5)$.

We next assessed whether bursts of synaptic activity alone (in the absence of any postsynaptic spiking) were sufficient to modulate synaptic strength. Repetitive bursts (10 s duration, $10 \mathrm{~s}$ interburst interval, repeated $10 \times$ ) of $10 \mathrm{~Hz}$ presynaptic stimulation were delivered while the postsynaptic membrane potential was maintained near $-60 \mathrm{mV}$ in current clamp with hyperpolarizing current injection to ensure EPSPs were subthreshold for the entire protocol (Fig. 2C). This presynaptic burst protocol, in the absence of postsynaptic spiking had no net effect on synaptic efficacy $($ EPSC at $0 \mathrm{~min}=96 \pm 16 \%$ of control; EPSC at $30 \mathrm{~min}=$ $114 \pm 28 \%$ of control, $p>0.05, n=6$ ) (Fig. $2 D$ ).

These data suggest that coincident epochs of postsynaptic spiking with glutamate receptor activation are necessary to drive efficient synaptic depression in VP neurons. By contrast, physiologically relevant bursts of synaptic activity in the absence of postsynaptic spikes fail to reliably affect synaptic strength.

\section{Mechanisms of synaptic depression}

To determine the locus of the depression mediated by pairing of bursts of presynaptic and postsynaptic activity (Fig. $2 \mathrm{~B}$ ), we analyzed the coefficient of variation (CV) of the evoked EPSC in control, immediately after burst pairing and $30 \mathrm{~min}$ later. There was a significant reduction in $1 / \mathrm{CV}^{2}$ after burst pairing $(p<0.05$, $n=5)$ that recovered to control levels by $30 \min (p>0.05, n=5)$ (Fig. $3 B$ ). This suggests that the inhibition is mediated by a decrease in glutamate release from the presynaptic terminal. To provide further evidence for this, we analyzed the frequency and amplitude of evoked asynchronous release events. Since glutamate synapses onto MNCs display robust asynchronous release in control conditions, $\mathrm{Sr}^{2+}$ or other manipulations to desynchronize the EPSC were not necessary (Iremonger and Bains, 2007). When individual asynchronous release events were analyzed (see Materials and Methods), burst pairing was found to significantly reduce the number of events immediately after pairing ( $62 \pm 4 \%$ of control at $0 \mathrm{~min}, p<0.05, n=5$ ) (Fig. $3 C$ ), which recovered to control levels after $30 \min (95 \pm 9 \%$ of control, $p>0.05, n=5$ ). This provides further support for a presynaptic locus of the inhibition. There was a small, but nonsignificant decrease in the amplitude of asynchronous release events after pairing ( $87 \pm 4 \%$ of control amplitude, $p>0.05, n=5$ ) (Fig. 3D). Together these data suggest that burst pairing induces a short-term synaptic depression that is expressed at a presynaptic locus.

Based on the findings that the burst pairing induced depression required both presynaptic and postsynaptic activity and this depression was expressed presynaptically, we hypothesized that the release of a retrograde messenger was necessary. One possibility is that the coincident activity at the glutamate synapses recruits postsynaptic mGluRs, resulting in the release of a retrograde signal. Many previous studies have demonstrated the necessity and sufficiency for activation of mGluRs in driving retrograde transmitter release (Maejima et al., 2001; Varma et al., 2001; Chevaleyre and Castillo, 2003). To determine whether ac- 

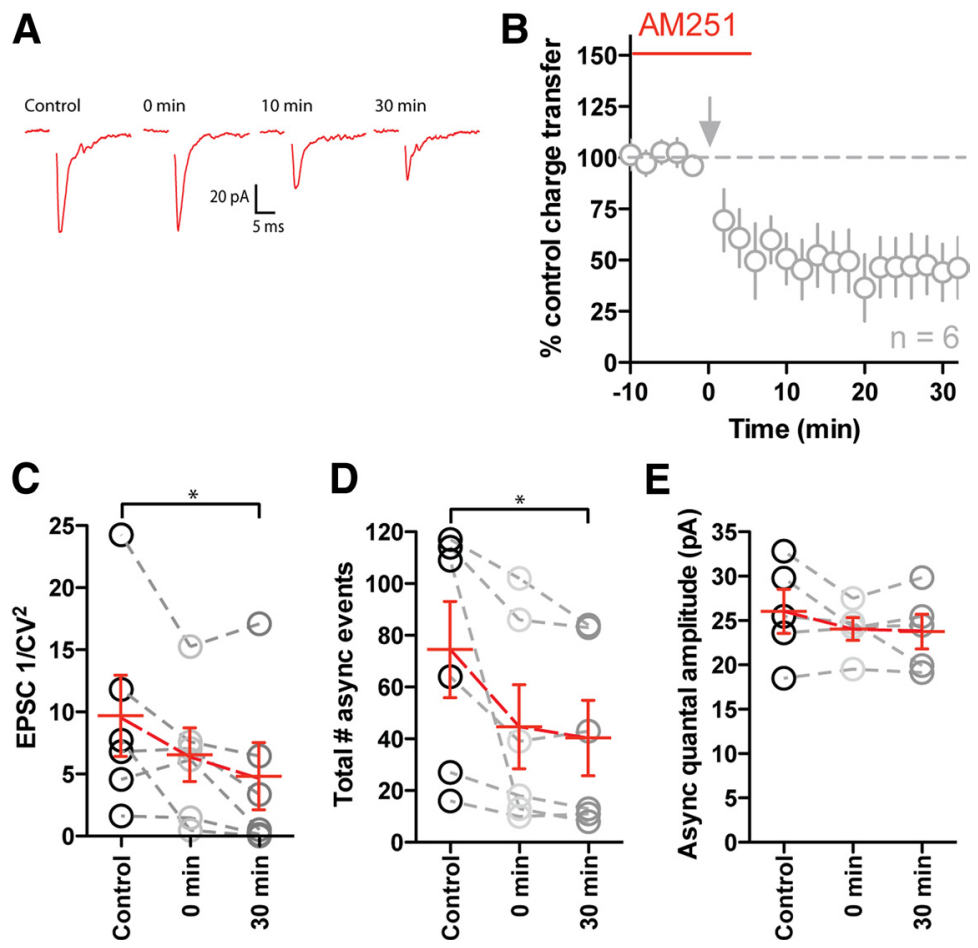

Figure 4. Blockade of $C B 1$ receptors alone unmasks a presynaptic LTD. A, Pairing of $10 \mathrm{~Hz}$ synaptic activity and spiking in the presence of the $(B 1$ receptor antagonist AM251 (5 $\mu \mathrm{m}$ ) induces long term depression of synaptic transmission (EPSC at $30 \mathrm{~min}=$ $46 \pm 15 \%$ of control, $p<0.05, n=6, \boldsymbol{B})$. C, This LTD is expressed presynaptically as it is associated with a significant change in $1 / \mathrm{CV}^{2}$ of the EPSC (Control vs $30 \mathrm{~min}, p<0.05, n=6$ ) and a reduction in the total number of asynchronously released quanta (Control vs $30 \mathrm{~min}, p<0.05, \boldsymbol{D}$ ). $\boldsymbol{E}$, There was no significant change in the amplitude of quantal asynchronous EPSCs after burst pairing in the presence of AM251 $(p>0.05, n=6) .{ }^{*} p<0.05$.
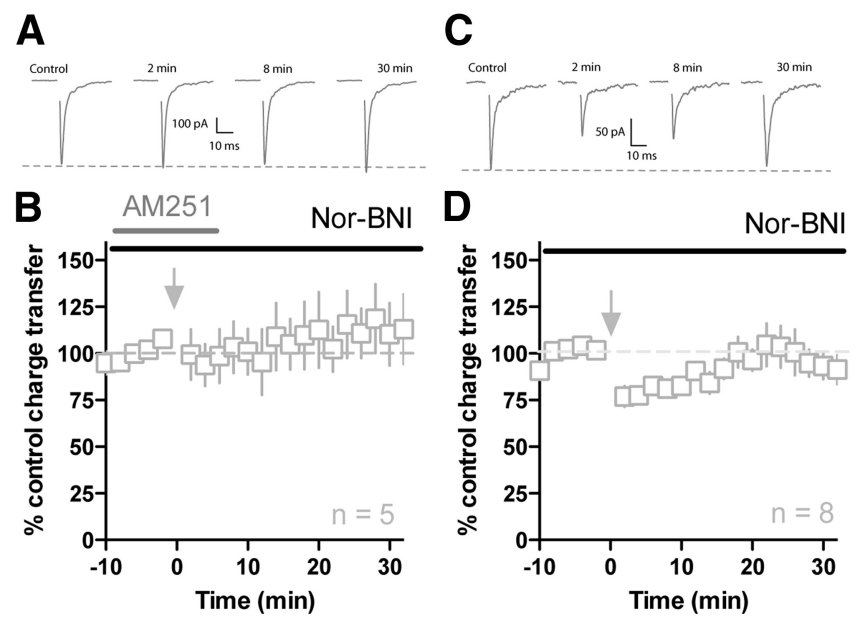

Figure 5. Synaptic depression requires both dynorphin and endocannabinoid signaling. $\boldsymbol{A}$, Representative traces (15 trial average) of EPSCs from control and various time points after burst pairing in the presence of both AM251 (5 $\mu \mathrm{m})$ and the $\kappa$-opioid receptor antagonist, nor-BNI (100 nM). $\boldsymbol{B}$, Burst pairing under these conditions failed to induce any synaptic depression (EPSC at 0 $\min =99 \pm 14 \%$ of control, $p>0.05, n=5$ ). C, Representative traces ( 15 trial average) of EPSCs from control and various time points after burst pairing in the presence of only nor-BNI (100 nm). $\boldsymbol{D}$, In the presence of nor-BNI, burst pairing still evokes a transient synaptic depression (EPSC at $0 \mathrm{~min}=77 \pm 6 \%$ of control, $n=8, p<0.05$; EPSC at $30 \mathrm{~min}=91 \pm 8 \%$ of control, $p>$ $0.05, n=8)$.

tivation of mGluRs is important for the synaptic depression observed here, we repeated the burst pairing protocol in the presence of the group I/II mGluR antagonist MCPG $(200 \mu \mathrm{M})$. Consistent with an essential role for mGluRs, MCPG blocked the induction of burst pairing depression (EPSC at $0 \mathrm{~min}=95 \pm$ $14 \%$ control, $p>0.05, n=5$ ) (Fig. $3 E$ ). To confirm that the
mGluRs are postsynaptic, we repeated the burst pairing protocol after dialyzing the postsynaptic cell with an internal solution containing GDP- $\beta \mathrm{s}(2 \mathrm{~mm})$. Inhibition of G-protein signaling exclusively in the postsynaptic neuron was sufficient to prevent burst pairing depression (EPSC at $0 \mathrm{~min}=122 \pm 13 \%$ control, $p>0.05$, $n=5$ ) (Fig. $3 F$ ).

\section{Burst pairing plasticity requires multiple retrograde transmitters}

Based on the observation that the burst pairing plasticity requires the activation of mGluRs and induces a presynaptic inhibition of transmitter release that lasts longer than that seen after postsynaptic spiking alone, we designed a series of experiments to identify the retrograde transmitter(s) involved.

Since mGluR activation can also liberate $\mathrm{eCBs}$, we repeated the burst pairing protocol in the presence of the CB1R antagonist AM251 (5 $\mu \mathrm{M})$. Remarkably, rather than blocking synaptic depression, burst pairing in the presence of AM251 induced LTD of synaptic transmission. Whereas in the absence of AM251, the EPSC charge had returned to baseline levels after $30 \mathrm{~min}$ (EPSC at $30 \mathrm{~min}$ : control pairing $=92 \pm 6 \%, n=5$ ), following burst pairing in AM251, the EPSC remained significantly depressed (EPSC at $30 \mathrm{~min}=46 \pm 15 \%$ of control, $p<0.05$, $n=6$ ) (Fig. $4 B$ ). We next examined the nature of the LTD more carefully. LTD was accompanied by a change in $1 / \mathrm{CV}^{2}$ of the EPSC (control $=9.46 \pm 3.27 ; 30$ $\min =4.59 \pm 2.70, p<0.05, n=6)$ and $a$ reduction in the total number of asynchronous quantal events (\# of async release events at $30 \mathrm{~min}=56 \pm 10 \%$ of control, $p<0.05, n=6$ ) with no change in asynchronous quantal amplitude (async amplitude at $30 \mathrm{~min}=93 \pm 6 \%$ of control, $p>0.05, n=6$ ) (Fig. 4C-E). This is consistent with a presynaptic locus of expression.

We have previously shown that dynorphin, released from the dendrites of VP neurons, induces presynaptic LTD in the presence of AM251 (Iremonger and Bains, 2009). To assess whether eCBs may be inhibiting dynorphin release or masking its effects, we repeated the pairing protocol in the presence of AM251 and nor-binaltorphimine (nor-BNI, $100 \mathrm{~nm}$ ), the antagonist of the $\kappa$-opioid receptor that binds dynorphin (Chavkin et al., 1982). Blockade of both CB1Rs and $\kappa$-opioid receptors completely blocked burst pairing-induced synaptic depression (EPSC at $0 \mathrm{~min}=99 \pm 14 \%$ of control, $p>0.05, n=5$ ) (Fig. $5 B$ ). When nor-BNI was administered alone, however, burst pairing still produced a significant but transient synaptic depres- 
sion (EPSC at $0 \mathrm{~min}=77 \pm 6 \%$ of control in nor-BNI, $p<0.05$; EPSC at $30 \mathrm{~min}=$ $91 \pm 8 \%$ of control, $p>0.05, n=8$ ) (Fig. $5 D)$. Based on these observations, we conclude that eCBs contribute to short-term depression at glutamate synapses on VP cells, while simultaneously preventing the development of long-term depression.

\section{CB1 receptor activation does not occlude $\kappa$-opioid presynaptic inhibition}

eCBs may impair LTD either by decreasing the release of dynorphin from the postsynaptic neuron or by limiting the actions of dynorphin at its presynaptic receptor. Several studies have demonstrated cross talk between the eCB and $\kappa$-opioid systems (Welch and Eads, 1999; Dogrul et al., 2002; Sáez-Cassanelli et al., 2007) in which activation of one receptor system alters the efficacy of the other. We have previously shown that dynorphin inhibits the EPSC via a presynaptic mechanism (Iremonger and Bains, 2009). In other central neurons, CB1 receptor-dependent synaptic inhibition is also presynaptically mediated (Kreitzer and Regehr, 2001; Wilson et al., 2001). Therefore, we tested whether there is cross talk between these two presynaptic receptors at glutamate terminals onto VP neurons.

First we confirmed the presynaptic effects of CB1 receptor activation in VP neurons. Bath application of the CB1R agonist WIN-55,212-2 (WIN, 2-3 $\mu \mathrm{M}$ ) reduced the EPSC to $48 \pm 7 \%$ of control after $20 \min (p<0.05, n=7)$. WIN also produced a significant decrease in the EPSC $1 / \mathrm{CV}^{2}(5.1 \pm 1.2$ control, $2.1 \pm 0.5$ after WIN, $p<0.05, n=7)$ and the number of asynchronous release events (39.9 \pm 6.7 control, $13.1 \pm 3.7$ after WIN, $p<0.05, n=7$ ) without changing the asynchronous quantal amplitude (21.8 \pm 3.4 pA control, $23.2 \pm 4.9$ pA after WIN, $p>0.05, n=7)$. This is consistent with previous reports that activation of CB1 receptors inhibits presynaptic glutamate release.

We next performed an occlusion experiment to test whether activation of presynaptic CB1 receptors can occlude the functioning of presynaptic $\kappa$-opioid receptors. In five cells, we followed the WIN application with a subsequent bath application of dynorphin-A (Fig. $6 \mathrm{~B}$ ). WIN reduced the EPSC to $56 \pm 7 \%$ of control in five cells $(p<0.05)$ (Fig. $6 B)$. Subsequent bath application of $1 \mu \mathrm{M}$ dynorphin A (1-17) further inhibited the EPSC to $22 \pm 8 \%$ of control after $10 \mathrm{~min}(n=5)$ (Fig. $6 B$ ). This inhibition by dynorphin A in the presence of WIN was not significantly different from the inhibition observed when dynorphin $\mathrm{A}$ is applied in isolation $(p>0.05, n=5$ ) (Fig. 6C,D). The inhibition by dynorphin in the presence of WIN was likely not due to actions at other opioid receptors, as application of the synthetic $\kappa$-opioid receptor agonist U69593 (200 nM, $5 \mathrm{~min}$ ) also pow-
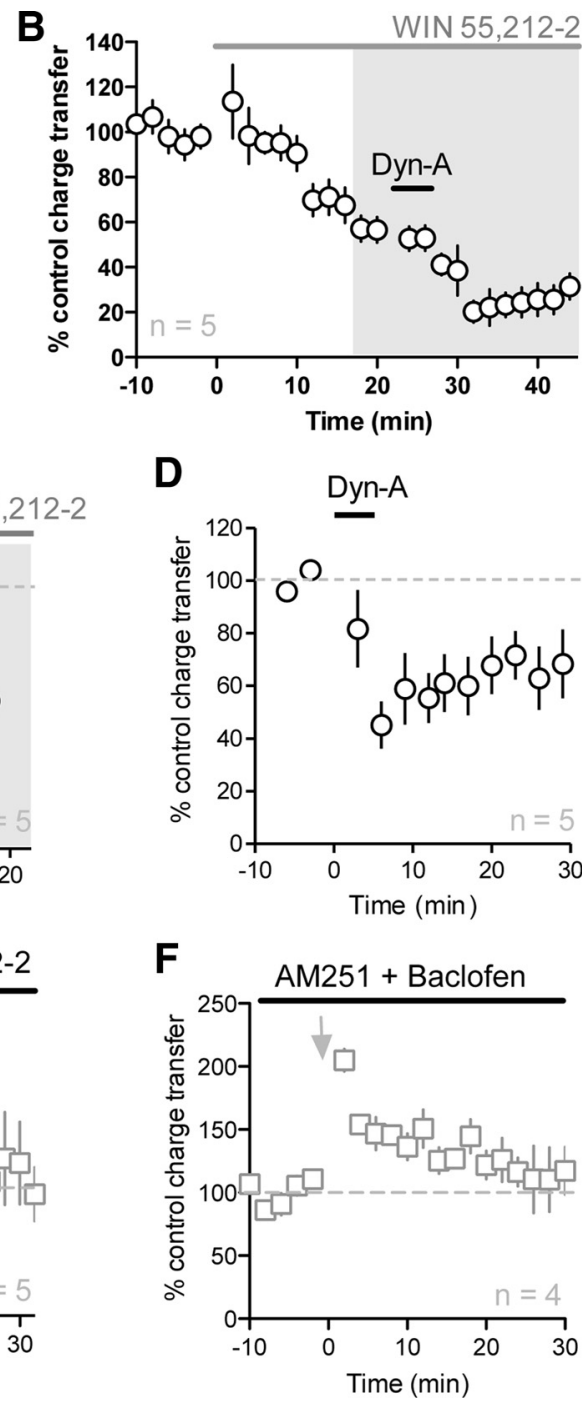

Figure 6. CB1 receptor activation regulates dynorphin release, not presynaptic $\kappa$-opioid signaling. $\boldsymbol{A}$, Representative traces (15 trial average) of EPSCs from control, after $3 \mu \mathrm{m}$ WIN 55,212-2 and WIN + Dynorphin A (1 $\mu \mathrm{m})$. B , Graph showing that Dynorphin $A$ is still able to inhibit the evoked EPSC after prior inhibition by WIN. Gray shaded area in graph is expanded in C. C, If the baseline

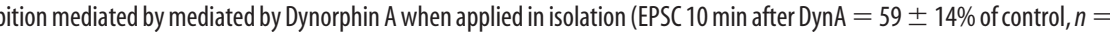
$7 \%$ of control at $0 \mathrm{~min}, p>0.05, n=5)$. $\boldsymbol{F}$, Inhibition of glutamate release probability with baclofen ( $300 \mathrm{~nm}$, in the presence of $5 \mu \mathrm{M}$ AM251) also prevents burst pairing depression (EPSC at $0 \mathrm{~min}=205 \pm 9 \%$ of control, $p>0.05, n=4$ ).

erfully inhibited the EPSC in the presence of WIN (EPSC $=$ $15 \pm 7 \%$ of WIN normalized baseline, $n=3$ ). Based on these data, we conclude that activation of CB1 receptors does not occlude the inhibition mediated by activation of $\kappa$-opioid receptors and instead that $\mathrm{CB} 1$ receptors may regulate the dendritic release of dynorphin.

Next we tested whether glutamate synapses onto VP neurons were under tonic CB1R-mediated inhibition. Bath-applied AM251 had a variable effect on the EPSC with some cells showing an enhancement of the EPSC, while others showed no change. Overall there was no significant effect of AM251 on the EPSC ( 15 min after AM251 EPSC $=261 \pm 95 \%$ of control charge transfer, $p>0.05, n=8$ ). The lack of a statistically significant change in the EPSC was likely due to the highly variable effect of AM251. However, even if cells were separated into responders and non- 
A
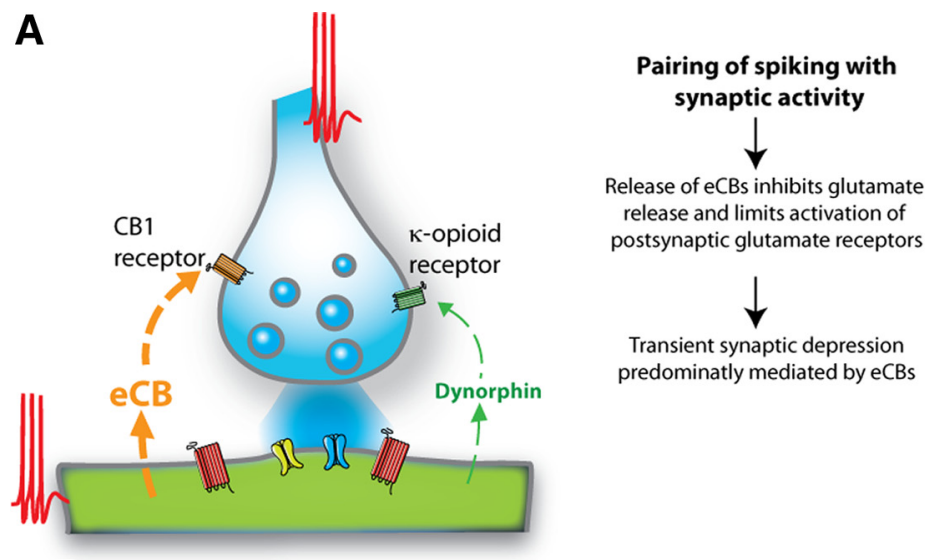

B

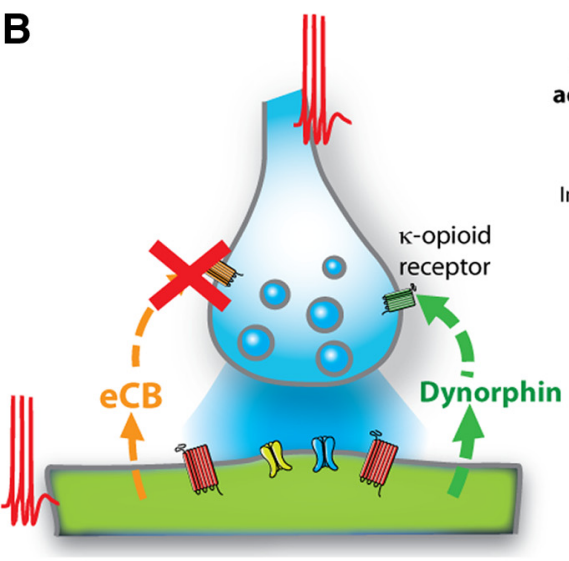

Pairing of spiking with synaptic activity with CB1 receptors blocked

Increased glutamate release due to loss of feedback inhibition mediated by eCBs somatodendritic release of dynorphin

Long term synaptic depression
Enhanced stimulus for the mediated by dynorphin

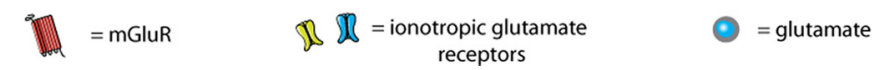

Figure 7. Proposed mechanisms controlling the magnitude of synaptic depression by coreleased retrograde transmitters. $\boldsymbol{A}$, Pairing of synaptic activity and spiking under control conditions induces the release of endocannabinoids (eCB) which feedback onto presynaptic glutamate terminals to inhibit presynaptic glutamate release via CB1 receptors. This synaptic depression is robust but transient. $\boldsymbol{B}$, When CB1 receptors are blocked, there is no longer fast feedback inhibition of glutamate release, thus allowing for more glutamate release during bursts of synaptic activity. Increased glutamate release will allow for enhanced postsynaptic glutamate receptor activation, which in turn drives the postsynaptic release of dynorphin. Dynorphin acts as a retrograde transmitter to induce a long-term depression of synaptic transmission.

responders, the variability in responses still prevented us from finding significant changes in response to AM251.

\section{Mechanisms regulating retrograde transmitter release}

Since CB1R activation does not occlude the actions of dynorphin, we hypothesized that eCBs may indirectly interfere with the release of dynorphin from the postsynaptic cell. As shown above, activation of CB1Rs depresses glutamate release. Since robust depression following paired synaptic stimulation and postsynaptic spiking requires the activation of postsynaptic mGluRs, we hypothesized that CB1Rs may decrease the probability of dynorphin release by lowering the presynaptic release of glutamate and thus limiting the activation of postsynaptic mGluRs.

To test this, we repeated the burst pairing protocol in the presence of the CB1 receptor agonist WIN $(1 \mu \mathrm{M})$. WIN, bath applied for a minimum of $30 \mathrm{~min}$ before the induction of burst pairing plasticity, depressed glutamate release (see above). In the presence of WIN, burst pairing now elicited a transient, nonsignificant potentiation of the EPSC $(187 \pm 37 \%$ of control at $0 \mathrm{~min}$, $p>0.05, n=5$ ) (Fig. $6 E$ ) that quickly returned to control levels. Thirty minutes after pairing, EPSC charge transfer was still not significantly different from baseline $(96 \pm 21 \%$ of control at 30 min, $p>0.05)$. The EPSC $1 / \mathrm{CV}^{2}$ also did not significantly change either immediately or $30 \mathrm{~min}$ after burst pairing in the presence of WIN $($ control $=3.3 \pm 1.2,0$ $\min =7.9 \pm 1.8,30 \min =3.6 \pm 1.7, p>$ $0.05, n=5)$. These data demonstrate a failure to induce burst-pairing depression when CB1Rs are tonically active.

Since we have shown that CB1R activation does not preclude the actions of dynorphin through $\kappa$-opioid receptors, we hypothesized that CB1R activation, by lowering glutamate release, may contribute to a situation that is not permissive of the release of dynorphin. To test whether the glutamate release probability was an important determinant for the induction of burst pairing depression, we used an independent method to lower probability of release at glutamate synapses. We (Kuzmiski et al., 2009) and others (Kombian et al., 1996; Kolaj et al., 2000) have shown that $\mathrm{GABA}_{\mathrm{B}}$ receptors are powerful inhibitors of glutamate release at synapses onto MNCs. Consequently, we repeated the burst pairing protocol in the presence AM251 and the $\mathrm{GABA}_{\mathrm{B}}$ receptor agonist baclofen (300 nM). Similar to the observations in the presence of WIN, we failed to induce synaptic depression following burst pairing in the presence of baclofen and AM251 (205 $\pm 9 \%$ of control at 0 min, $p>0.05, n=4$ ) (Fig. $6 F$ ). There was no significant change in the EPSC $1 / \mathrm{CV}^{2}$ after this burst pairing protocol either (control $=5.4 \pm 0.8,0 \mathrm{~min}=9.4 \pm 2.5$, $30 \min =6.5 \pm 1.1, p>0.05, n=4)$.

These results indicate that reducing presynaptic glutamate release and subsequent postsynaptic mGluR activation abolishes burst pairing depression. Together, our data suggest two things: First, the duration and magnitude of synaptic depression observed is dependent on both the level of glutamate receptor activation and postsynaptic spiking. Second, eCBs inhibit dynorphin release indirectly by lowering probability of release at glutamate synapses and thus reducing the activation of postsynaptic metabotropic glutamate receptors. A schematic illustrating these points is shown in Figure 7.

\section{Discussion}

Here we report a novel form of synaptic plasticity in which one retrograde signal, eCB, regulates the release of a second retrograde signal, dynorphin, thus determining the magnitude and duration of synaptic depression. eCBs do not directly impair dynorphin release, nor do they interfere with signaling at $\kappa$-opioid receptors, but rather decrease mGluR activation by lowering glutamate release probability. By preventing the feedforward recruitment of dynorphin, eCBs ensure that synaptic depression is temporary. This rapid recovery from depression may be particularly important for a neural system that must remain in a vigilant state to ensure appropriate regulation of an organism's internal environment. 
We have shown that bursts of postsynaptic spikes, in the absence of presynaptic stimulation, induced a short-lasting synaptic depression mediated by eCB release. When postsynaptic spiking was paired with presynaptic stimulation, however, we observed a robust but reversible synaptic depression that was mediated by the corelease of eCBs and dynorphin. In contrast, presynaptic stimulation, in the absence of postsynaptic spiking, failed to induce reliable changes in synaptic strength, indicating a requirement for coincident activation for presynaptic and postsynaptic elements. Blockade of CB1Rs unmasked LTD that failed to recover. LTD could be prevented either by blocking both CB1 and $\kappa$-opioid receptors or by lowering glutamate release probability before the burst pairing induction protocol. The impact of combined presynaptic and postsynaptic activity on synaptic function will therefore depend on the initial release probability of the synapse, which, immediately following a burst of activity, is controlled by eCBs.

We have demonstrated that the magnitude of retrograde synaptic depression is regulated by indirect but not direct cross talk between the two retrograde signals. Specifically, activation of CB1 receptors had no effect on the ability of exogenous dynorphin to inhibit glutamate release. Instead, $\mathrm{eCBs}$ released from the postsynaptic neuron decrease glutamate release, lower the amount of postsynaptic mGluR activation, and subsequently decrease the probability of dynorphin release. Consequently, when CB1 receptors are blocked and glutamate release is allowed to build, this augments dynorphin release, unmasking a more robust and longer-lasting depression than seen in control conditions. The ability of eCBs to prevent LTD through modulation of dynorphin signaling suggests a new role for eCBs in the regulation of synaptic function. While there are several examples in the CNS of neurons releasing multiple retrograde signaling molecules in response to different stimulation paradigms, we are aware of only a few studies where retrograde transmitters are simultaneously released from a neuron in response to a single stimulation protocol (Duguid and Smart, 2004; Hirasawa et al., 2004; Di et al., 2009). To the best of our knowledge, ours is the first demonstration that the effects of one coreleased retrograde transmitter negatively impact the release of a second retrograde signal.

Several lines of evidence suggest that activation of presynaptic $\mathrm{CB} 1$ receptors and the subsequent reduction in glutamate release are responsible for the effects of eCBs on dynorphin signaling. First, we ruled out the possibility that activation of presynaptic CB1 receptors modifies signaling through presynaptic $\kappa$-opioid receptors. Specifically, we performed the experiment where the $\mathrm{CB} 1$ receptor agonist WIN was bath applied, and after reaching a stable baseline of inhibition, we then applied dynorphin A. In these experiments, dynorphin A further inhibited the EPSC. This inhibition was not different from that observed with dynorphin A alone. These data suggest that activation of CB1 receptors does not affect signaling through the $\kappa$-opioid receptor. Second, pairing of synaptic activity and spiking induced LTD in the presence of the CB1 receptor antagonist AM251; depression, however, was not observed when the same pairing protocol was induced in the presence of WIN. Since WIN inhibits single EPSCs onto VP neurons by $\sim 50 \%$, the extent of postsynaptic glutamate receptor activation during the burst pairing protocol would be dramatically reduced. Inhibiting glutamate release with the $\mathrm{GABA}_{\mathrm{B}}$ agonist baclofen produced effects similar to that of WIN. Finally, consistent with the proposed importance of glutamate receptor activation for eCB and dynorphin release, the mGluR antagonist MCPG blocked the synaptic depression mediated by burst pair- ing. A similar indirect modulation of retrograde transmitter release, but in this case by a feedforward, associative signal, has recently been reported in the cerebellum (Carey and Regehr, 2009).

One alternative possibility is that VP neurons possess postsynaptic CB1 receptors and that dendritically released eCBs have an autocrine effect that directly inhibits the release of large dense core vesicles from the postsynaptic neuron. Several pieces of data, however, argue against this hypothesis. First, MNCs appear to express CB1 receptors only on presynaptic nerve terminals and not on their postsynaptic membranes (Hirasawa et al., 2004). Second, other studies demonstrating the presence of functional postsynaptic CB1 receptors have shown that bath application of $\mathrm{CB} 1$ receptor agonists can induce an outward $\mathrm{K}^{+}$current (Kreitzer et al., 2002; Bacci et al., 2004). We, however, failed to observe either an outward current or a change in membrane resistance following application of WIN even at high doses $(6 \mu \mathrm{M})$ (data not shown). While only the synaptic terminals onto MNCs appear to express CB1 receptors, several studies have shown $\kappa$-opioid receptors to be both presynaptically and postsynaptically located in MNCs (Shuster et al., 2000; Brown and Bourque, 2004; Honda et al., 2004). We have previously shown that there is no postsynaptic glutamate receptor inhibition with bath-applied $\kappa$-opioid receptor agonists (Iremonger and Bains, 2009); however, this does not rule out that postsynaptic $\kappa$-opioid receptors are being activated in response to the different burst firing protocols used in this current study. Finally, consistent with our proposed mechanisms driving retrograde transmitter release, mGluR1 is also postsynaptically expressed in PVN magnocellular neurons (Shigemoto et al., 1992; Mateos et al., 1998).

It is interesting to note that there does not appear to be any "cross talk" between presynaptic $\kappa$-opioid and CB1 receptors. While both of these receptors are $\mathrm{G}_{\mathrm{i} / \mathrm{o}}$ coupled and inhibit transmitter release, they likely act through different mechanisms at presynaptic terminals in the PVN. CB1-dependent synaptic depression is classically due to inhibition of presynaptic voltagegated $\mathrm{Ca}^{2+}$ channels (Kreitzer and Regehr, 2001; Wilson et al., 2001). $\kappa$-Opioid-dependent presynaptic depression, however, does not result from inhibition of presynaptic $\mathrm{Ca}^{2+}$ channels but is instead likely due to a direct inhibition of vesicle fusion (Iremonger and Bains, 2009). These divergent inhibitory pathways would allow for cumulative synaptic inhibition when both CB1 and $\kappa$-opioid receptors were activated.

One alternative explanation is that presynaptic terminals onto VP neurons express only one type of receptor and not both CB1 and $\kappa$-opioid receptors together. We think this is unlikely because synaptic responses in all cells tested were uniformly inhibited by bath-applied WIN or $\kappa$-opioid receptor agonists.

In our previous study, we showed that somatodendritic dynorphin release could be evoked with repetitive strong postsynaptic depolarizations (to $0 \mathrm{mV}$ ) without coincident synaptic stimulation (Iremonger and Bains, 2009). These experiments were performed in the presence of AM251 to block eCB signaling and amastatin to inhibit dynorphin breakdown in the synaptic cleft. This protocol was used to study dynorphin signaling without any cofounding eCB effects. Observations from in vivo experiments, however, indicate the frequency of synaptic inputs onto MNCs is high (Bourque and Renaud, 1991). In addition, phasic bursting in VP neurons requires glutamatergic synaptic activity in vivo (Nissen et al., 1995; Brown et al., 2004). Therefore, to more accurately mimic in vivo conditions, the stimulation protocol used in this current study paired both trains of EPSPs with postsynaptic spiking. Because the release of large dense core vesicles 
from the dendrites of MNCs is greatly facilitated by $\mathrm{Ca}^{2+}$ release from internal stores (Ludwig et al., 2002), the combination of postsynaptic spiking and mGluR activation would likely be a very effective stimulus to drive somatodendritic dynorphin release. As the actions of group I mGluRs are often potentiated by increases in postsynaptic $\mathrm{Ca}^{2+}$ (Huang et al., 2004), this is also a likely reason why pairing of synaptic activity and spiking proved such an effective stimulus for driving retrograde transmitter release.

Why might multiple retrograde messengers released from a single neuron be important for the regulation of excitability? Our results demonstrate that different retrograde transmitters require different intensities of postsynaptic activation to be efficiently released. In this case, moderate neuronal activation (spiking alone) will primarily release eCBs to affect glutamate release probability. Since glutamate release is reduced, the retrogradely acting eCBs will also lower the probability that dynorphin will subsequently be released. Thus, only under very intense periods of postsynaptic activation would retrograde dynorphin signaling be recruited.

Previous studies have demonstrated that persistent activation of $\mathrm{CB} 1$ receptors can transform a transient synaptic depression into LTD (Chevaleyre and Castillo, 2003). Why then would a synapse require multiple retrograde transmitters if eCBs are capable of producing depression over variable time scales? One possibility is that both the pattern of activation in addition to the duration and intensity of activation are key determinants in driving the release of specific retrograde transmitters. This would afford the postsynaptic neuron a great deal of flexibility by ensuring that release of particular substances is tuned to defined activity patterns.

Neurons are endowed with multiple mechanisms to regulate their excitability. Here we have demonstrated that multiple retrograde messengers can be released from a single neuron in response to physiologically relevant bursting activity. These retrograde signals interact with one another to dynamically regulate the synaptic plasticity induced. This suggests that excitatory synapses not only possess different feedback mechanisms that operate over varying activity levels, but also that this feedback inhibition can be tuned by coreleased transmitters.

\section{References}

Bacci A, Huguenard JR, Prince DA (2004) Long-lasting self-inhibition of neocortical interneurons mediated by endocannabinoids. Nature 431:312-316.

Bains JS, Ferguson AV (1997) Nitric oxide regulates NMDA-driven GABAergic inputs to type I neurones of the rat paraventricular nucleus. J Physiol 499:733-746.

Bourque CW, Renaud LP (1991) Membrane properties of rat magnocellular neuroendocrine cells in vivo. Brain Res 540:349-352.

Brown CH, Bourque CW (2004) Autocrine feedback inhibition of plateau potentials terminates phasic bursts in magnocellular neurosecretory cells of the rat supraoptic nucleus. J Physiol 557:949-960.

Brown CH, Bull PM, Bourque CW (2004) Phasic bursts in rat magnocellular neurosecretory cells are not intrinsically regenerative in vivo. Eur J Neurosci 19:2977-2983.

Carey MR, Regehr WG (2009) Noradrenergic control of associative synaptic plasticity by selective modulation of instructive signals. Neuron $62: 112-122$

Chavkin C, James IF, Goldstein A (1982) Dynorphin is a specific endogenous ligand of the kappa opioid receptor. Science 215:413-415.

Chevaleyre V, Castillo PE (2003) Heterosynaptic LTD of hippocampal GABAergic synapses: a novel role of endocannabinoids in regulating excitability. Neuron 38:461-472.

Di S, Maxson MM, Franco A, Tasker JG (2009) Glucocorticoids regulate glutamate and GABA synapse-specific retrograde transmission via divergent nongenomic signaling pathways. J Neurosci 29:393-401.
Dogrul A, Gardell LR, Ma S, Ossipov MH, Porreca F, Lai J (2002) 'Knockdown' of spinal CB1 receptors produces abnormal pain and elevates spinal dynorphin content in mice. Pain 100:203-209.

Duguid IC, Smart TG (2004) Retrograde activation of presynaptic NMDA receptors enhances GABA release at cerebellar interneuron-Purkinje cell synapses. Nat Neurosci 7:525-533.

Hirasawa M, Schwab Y, Natah S, Hillard CJ, Mackie K, Sharkey KA, Pittman QJ (2004) Dendritically released transmitters cooperate via autocrine and retrograde actions to inhibit afferent excitation in rat brain. J Physiol 559:611-624.

Honda E, Ono K, Inenaga K (2004) DAMGO suppresses both excitatory and inhibitory synaptic transmission in supraoptic neurones of mouse hypothalamic slice preparations. J Neuroendocrinol 16:198-207.

Huang YH, Sinha SR, Tanaka K, Rothstein JD, Bergles DE (2004) Astrocyte glutamate transporters regulate metabotropic glutamate receptormediated excitation of hippocampal interneurons. J Neurosci 24:45514559 .

Iremonger KJ, Bains JS (2007) Integration of asynchronously released quanta prolongs the postsynaptic spike window. J Neurosci 27:66846691.

Iremonger KJ, Bains JS (2009) Retrograde opioid signaling regulates glutamatergic transmission in the hypothalamus. J Neurosci 29:7349-7358.

Jo YH, Schlichter R (1999) Synaptic corelease of ATP and GABA in cultured spinal neurons. Nat Neurosci 2:241-245.

Jonas P, Bischofberger J, Sandkühler J (1998) Corelease of two fast neurotransmitters at a central synapse. Science 281:419-424.

Kolaj M, Yang CR, Renaud LP (2000) Presynaptic GABA(B) receptors modulate organum vasculosum lamina terminalis-evoked postsynaptic currents in rat hypothalamic supraoptic neurons. Neuroscience 98:129-133.

Kombian SB, Zidichouski JA, Pittman QJ (1996) GABAB receptors presynaptically modulate excitatory synaptic transmission in the rat supraoptic nucleus in vitro. J Neurophysiol 76:1166-1179.

Kreitzer AC, Regehr WG (2001) Retrograde inhibition of presynaptic calcium influx by endogenous cannabinoids at excitatory synapses onto Purkinje cells. Neuron 29:717-727.

Kreitzer AC, Carter AG, Regehr WG (2002) Inhibition of interneuron firing extends the spread of endocannabinoid signaling in the cerebellum. Neuron 34:787-796.

Kupfermann I (1991) Functional studies of cotransmission. Physiol Rev $71: 683-732$.

Kuzmiski JB, Pittman QJ, Bains JS (2009) Metaplasticity of hypothalamic synapses following in vivo challenge. Neuron 62:839-849.

Lu T, Rubio ME, Trussell LO (2008) Glycinergic transmission shaped by the corelease of GABA in a mammalian auditory synapse. Neuron 57:524-535.

Ludwig M, Sabatier N, Bull PM, Landgraf R, Dayanithi G, Leng G (2002) Intracellular calcium stores regulate activity-dependent neuropeptide release from dendrites. Nature 418:85-89.

Ludwig M, Bull PM, Tobin VA, Sabatier N, Landgraf R, Dayanithi G, Leng G (2005) Regulation of activity-dependent dendritic vasopressin release from rat supraoptic neurones. J Physiol 564:515-522.

Luther JA, Tasker JG (2000) Voltage-gated currents distinguish parvocellular from magnocellular neurones in the rat hypothalamic paraventricular nucleus. J Physiol 523:193-209.

Maejima T, Hashimoto K, Yoshida T, Aiba A, Kano M (2001) Presynaptic inhibition caused by retrograde signal from metabotropic glutamate to cannabinoid receptors. Neuron 31:463-475.

Maejima T, Oka S, Hashimotodani Y, Ohno-Shosaku T, Aiba A, Wu D, Waku K, Sugiura T, Kano M (2005) Synaptically driven endocannabinoid release requires $\mathrm{Ca} 2+$-assisted metabotropic glutamate receptor subtype 1 to phospholipase Cbeta4 signaling cascade in the cerebellum. J Neurosci 25:6826-6835

Mateos JM, Azkue J, Benítez R, Sarría R, Losada J, Conquet F, Ferraguti F, Kuhn R, Knöpfel T, Grandes P (1998) Immunocytochemical localization of the mGluR1b metabotropic glutamate receptor in the rat hypothalamus. J Comp Neurol 390:225-233.

Nissen R, Hu B, Renaud LP (1995) Regulation of spontaneous phasic firing of rat supraoptic vasopressin neurones in vivo by glutamate receptors. J Physiol 484:415-424.

Regehr WG, Carey MR, Best AR (2009) Activity-dependent regulation of synapses by retrograde messengers. Neuron 63:154-170.

Sáez-Cassanelli JL, Fontanella GH, Delgado-García JM, Carrión AM (2007) 
Functional blockage of the cannabinoid receptor type 1 evokes a kappaopiate-dependent analgesia. J Neurochem 103:2629-2639.

Shigemoto R, Nakanishi S, Mizuno N (1992) Distribution of the mRNA for a metabotropic glutamate receptor (mGluR1) in the central nervous system: an in situ hybridization study in adult and developing rat. J Comp Neurol 322:121-135.

Shuster SJ, Riedl M, Li X, Vulchanova L, Elde R (2000) The kappa opioid receptor and dynorphin co-localize in vasopressin magnocellular neurosecretory neurons in guinea-pig hypothalamus. Neuroscience 96:373383.

Tecuapetla F, Patel JC, Xenias H, English D, Tadros I, Shah F, Berlin J, Deisseroth K, Rice ME, Tepper JM, Koos T (2010) Glutamatergic signaling by mesolimbic dopamine neurons in the nucleus accumbens. J Neurosci 30:7105-7110.

Ueta Y, Fujihara H, Serino R, Dayanithi G, Ozawa H, Matsuda K, Kawata M, Yamada J, Ueno S, Fukuda A, Murphy D (2005) Transgenic expression of enhanced green fluorescent protein enables direct visualization for physiological studies of vasopressin neurons and isolated nerve terminals of the rat. Endocrinology 146:406-413.

Varma N, Carlson GC, Ledent C, Alger BE (2001) Metabotropic glutamate receptors drive the endocannabinoid system in hippocampus. J Neurosci 21:RC188.

Weisskopf MG, Zalutsky RA, Nicoll RA (1993) The opioid peptide dynorphin mediates heterosynaptic depression of hippocampal mossy fibre synapses and modulates long-term potentiation. Nature 362:423-427.

Welch SP, Eads M (1999) Synergistic interactions of endogenous opioids and cannabinoid systems. Brain Res 848:183-190.

Wilson RI, Kunos G, Nicoll RA (2001) Presynaptic specificity of endocannabinoid signaling in the hippocampus. Neuron 31:453-462.

Zilberter Y, Harkany T, Holmgren CD (2005) Dendritic release of retrograde messengers controls synaptic transmission in local neocortical networks. Neuroscientist 11:334-344. 